\title{
LA WEBQUEST: UNA ESTRATEGIA DIDÁCTICA EN EL AULA PARA LA ENSEÑANZA-APRENDIZAJE DEL PATRIMONIO CULTURAL
}

\author{
WEBQUEST: A TEACHING STRATEGY IN THE CLASSROOM FOR \\ THE LEARNING AND TEACHING OF CULTURAL HERITAGE
}

\author{
María de la Encarnación Cambil Hernández; ncambil@ugr.es \\ Guadalupe Romero Sánchez; guadalupers@ugr.es \\ Universidad de Granada
}

\section{RESUMEN}

El valor educativo del Patrimonio Cultural es un hecho cada vez más reconocido, sin embargo su tratamiento curricular en las diferentes etapas educativas es prácticamente anecdótico. Esta realidad provoca que en su formación inicial los maestros/as del Grado de Educación Primaria encuentren dificultades para lograr el aprendizaje significativo de los contenidos de la materia "Patrimonio Histórico y Cultural y su Didáctica". En este trabajo mostraremos una propuesta metodológica cuyo objetivo ha sido facilitar a los alumnos/as, desde la calidad y la innovación, la enseñanza-aprendizaje de la materia citada en la que hemos utilizado como metodología la observación directa y el trabajo por proyectos, complementada con la utilización de la WebQuest como estrategia didáctica.

PALABRAS CLAVE: Patrimonio Cultural, WebQuest, Innovación, observación directa, experiencia docente.

\begin{abstract}
The educational value of Cultural Heritage is an increasingly recognized. However its curricular treatment in different educational stages is almost anecdotal. This reality means that in their initial training teachers / as Grade Primary Education encounter difficulties achieves meaningful learning of the contents of the subject "Historical and Cultural Heritage and Teaching". In this work we show a methodology whose objective was to provide students / as, from the quality and innovation, teaching and learning of the subject cited in methodology we used as direct observation and project work, complemented by the use of the WebQuest as a teaching strategy.
\end{abstract}

KEYWORDS: Cultural Heritage, WebQuest, Innovation, Direct Observation, Teaching Experience. 


\section{INTRODUCCIÓN}

La experiencia docente que presentamos es fruto de la reflexión llevada a cabo sobre las dificultades de la enseñanza-aprendizaje de la materia "Patrimonio Histórico Cultural y su didáctica" que se imparte en el segundo semestre del primer curso del Grado de Maestro en Educación Primaria de la Universidad de Granada durante los años 2010 a 2013. Pero antes de analizar nuestra experiencia pasamos a exponer algunos aspectos que consideramos fundamentales.

El patrimonio cultural, fundamentalmente el patrimonio histórico artístico, ha sido utilizado tradicionalmente como recurso didáctico de manera transversal en diferentes áreas de conocimiento, no obstante, al igual que se ha avanzado en estrategias educativas también se ha hecho lo propio con el concepto de patrimonio y los elementos que lo integran ${ }^{1}$, por lo que, inevitablemente estos elementos deben ser tenidos en el cuenta en la planificación docente. Además, su incuestionable valor educativo se ha visto reforzado con la aprobación en 2013 del Plan Nacional de Educación y Patrimonio, con el que desde el Ministerio de Educación, Cultura y Deporte se quiere favorecer la inclusión paulatina de contenidos patrimonialistas en el currículo de todas las etapas educativas, entre otros elementos de interés y que consideramos todo un acierto ${ }^{2}$. La Universidad de Granada ha demostrado ser pionera en este sentido incluyendo tras la implementación del Plan Bolonia en el Plan de Estudios del Grado de Maestro en Educación Primaria, en el área de Didáctica de las Ciencias Sociales, la materia obligatoria mencionada en la que impartimos docencia.

\section{PUNTO DE PARTIDA}

Nuestra experiencia tuvo como punto de partida el análisis de la presencia en el currículo de primaria de contenidos relacionados con el patrimonio cultural. En diferentes leyes tanto de ámbito nacional como autonómico se señala la importancia del patrimonio y de su valor educativo, pero la realidad es que en el ámbito de la educación formal apenas hay contenidos patrimoniales, como quedó patente.

En la Ley Orgánica de Educación, en el Real Decreto $1513^{3}$, se organiza el currículo de Primaria en las siguientes áreas de conocimiento:

\footnotetext{
${ }^{1}$ Convención para el Patrimonio Cultural de México 1982:

Hhttp://www.ilam.org/index.php/es/talleres/materiales-apoyo/143-talleres-ilam/materiales-apoyo/288-patrimoniodefiniciones $H$. Consultado el 2/02/2105.

${ }^{2}$ En el texto del Plan queda recogida la importancia que adquiere a día de hoy el binomio Patrimonio y Educación o lo que vendría a ser la educación patrimonial para la correcta conservación del patrimonio para las generaciones futuros abriendo un horizonte de sostenibilidad que de otra manera sería difícil alcanzar. Por ello, el plan contempla la actuación en la educación formal a través de tres vías: la mejora de los materiales didácticos, la incorporación en el currículo de manera paulatina de mayores contenidos patrimoniales y la mejora de la formación de los docente en materia de patrimonio, como garantía de éxito. Instituto del Patrimonio Cultural de España:

Hhttp://ipce.mcu.es/conservacion/planesnacionales/educacion.htmlH. Consultado: 2/02/2015.

3 Debemos tener en cuenta que durante el desarrollo de nuestra experiencia aún no se había implantado en Andalucía la LOMCE.
} 
- Conocimiento del Medio Natural Social y Cultural

- Educación Física

- Educación artística

- Lengua castellana y literatura y si la hubiere lengua cooficial y literatura.

- Lengua extranjera

- Matemáticas

Como vemos no existe un área específica de patrimonio, los contenidos de Ciencias Sociales se integran, como consideramos lógico, con los de Ciencias Naturales en la primera de estas áreas, la cual se organiza en siete bloques temáticos: El entorno y su conservación; diversidad de los seres vivos; la salud y el desarrollo personal; personas, culturas y organización social; cambios en el tiempo; materia y energía y objetos, máquinas y tecnologías. Los aspectos relacionados con el patrimonio cultural se encuentran fundamentalmente en los bloques 4 y 5 , teniendo una presencia transversal y poco clara en el resto.

A nivel autonómico el patrimonio tiene mayor presencia, de hecho en la ley de agosto de 2007 (LEA) los contenidos del mismo área de conocimiento se organizan en ocho núcleos temáticos de los cuales el tercero es íntegramente de patrimonio: La construcción histórica, social y cultural de Andalucía; paisajes andaluces; el patrimonio en Andalucía; igualdad, convivencia e interculturalidad; salud y bienestar; progreso tecnológico y modelos de desarrollo; el uso responsable de los recursos y la incidencia de la actividad humana en el medio ${ }^{4}$.

A pesar de su presencia en el currículo, afortunadamente el patrimonio cultural por su valor educativo ha pasado de ser utilizado como un recurso a ser considerado una fuente primaria para la enseñanza-aprendizaje de las de las Ciencias Sociales ${ }^{5}$. Su docencia debe abordarse considerando su carácter multidisciplinar desde la Geografía (por la importancia del medio físico), desde la Historia (para la comprensión de los parámetros temporales) y desde la Historia del Arte, teniendo en cuenta además las aportaciones de la Antropología, el Derecho y la Economía, entre otros.

\section{ELABORACIÓN DE LA PROPUESTA: METODOLOGÍA}

Queríamos elaborar una propuesta docente innovadora que redundara en la calidad de la enseñanza. Para su elaboración siguiendo a Gallego y Mata (2005, pp. 162-166), tuvimos en cuenta la finalidad que perseguíamos, la adecuación al alumnado, al contexto y a los contenidos que íbamos a trabajar. En cuanto a la finalidad debía estar en relación con los objetivos fijados, respecto al alumnado, partiendo de los conocimientos previos debíamos favorecer la enseñanza-aprendizaje de la materia para facilitar que adquirieran las competencias fijadas y lograrán el aprendizaje

\footnotetext{
${ }^{4}$ Ley Andaluza de Educación:

Hhttp://www.juntadeandalucia.es/educacion/portal/com/bin/Contenidos/OEE/evaluacion/20141110_ProyDecretOrdenEducaPri maria/1415625250228_proyecto_deorden.pdfH. Consultado: 2/02/2015.

${ }^{5}$ Tal como señala Neus González, utilizar el patrimonio cultural como un recurso educativo puede ayudar a crear conciencia histórica en los jóvenes, ya que su aprendizaje les permitirá comprender mejor el pasado que los rodea, valorar la historicidad del presente y participar conscientemente en la construcción de su futuro personal y social (González, 2008:24), a lo que añadimos que no sólo como recurso sino también como fuente primaria.
} 
significativo de los contenidos a trabajar. La adecuación al contexto se haría teniendo en cuenta las condiciones en las que se desarrollaba el aprendizaje y los contenidos estaban condicionados por su carácter multidisciplinar y globalizado.

El análisis de la escasa presencia del patrimonio en el currículo nos llevó a la conclusión de que en ello radicaban la mayor parte de las dificultades que existen en el aula para abordar la enseñanza-aprendizaje de esta materia. Dificultades que comprobamos que no provenían sólo del alumnado, sino también del profesorado.

Respecto al alumnado nos encontramos que los futuros/as maestros/as llegan a la Universidad con conocimientos muy escasos de geografía, historia e historia del arte imprescindibles para nuestra materia ${ }^{6}$, este desconocimiento provoca una actitud acrítica respecto a temas de gran importancia histórica, social y cultural de los que son una consecuencia nuestro patrimonio. A eso se añade la visión restrictiva que tienen del patrimonio que les hace asimilar los contenidos de esta materia con actividades de ocio, turismo o elementos monumentales. Todo ello les impide tener una visión global del mismo y les lleva a considerar esta disciplina como algo aburrido y alejado de los intereses de los niños/as y de su comprensión ${ }^{7}$.

En cuanto al profesorado, en general, presenta carencias formativas en este campo. El carácter multidisciplinar de esta materia exige conocimientos muy amplios y requiere una profunda reflexión epistemológica de las disciplinas implicadas, sin ella se tendrá una visión restrictiva asimilándola a elementos monumentales y/o artísticos, sin tener en cuenta el patrimonio natural, el inmaterial o las nuevas figuras como las zonas patrimoniales que hoy forman parte de nuestro patrimonio cultural o viceversa.

A estas dificultades se añaden otras que parten de la organización del Plan de Estudios de este grado en nuestra universidad, ya que lo recomendable sería cursar esta materia en los últimos años de carrera o al menos en tercer curso, garantizando que los estudiantes hayan cursado ya la materia "Didáctica de las Ciencias Sociales" de segundo, que consideramos básica pode abordar con posterioridad la enseñanzaaprendizaje del patrimonio.

Ahora bien, volviendo a la metodología, el primer paso fue establecer los objetivos generales:

- Mantener una actitud crítica y autónoma en relación a los saberes, valores y prácticas que promueven las instituciones sociales, valorando especialmente el papel de la ciencia y la tecnología en la sociedad, así como, la importancia de una solida formación.

- Trabajar en equipo.

- Investigar y seguir aprendiendo con autonomía.

- Conocer y aplicar en las actividades del aula, las tecnologías de la información y la comunicación.

\footnotetext{
${ }^{6}$ La falta de formación en contenidos de estas disciplinas podemos decir que es general, con la excepción de los alumnos que cursaron Historia del Arte en Bachillerato, o una minoría a los que les gusta la Historia y han seguido trabajando sobre ello.

${ }^{7}$ Se da por supuesto que los niños y niñas perciben los concepto de espacio y tiempo en función de su madurez psicológica y no se tiene en cuenta que en la actualidad la situación ha cambiado ya que la televisión y las nuevas tecnologias son un estímulo que ha mejorado las capacidades infantiles para percibir el tiempo y el espacio en edades más tempranas.
} 
- Discernir selectivamente la información que contribuya al aprendizaje, a la formación cívica y a la riqueza cultural humanística.

- Innovar con creatividad.

En cuanto a los objetivos específicos de la materia del "Patrimonio Histórico y Cultura y su didáctica" destacamos:

- Comprender que el patrimonio es una construcción social que corresponde a un momento espacio-temporal concreto.

- Conocer los distintos elementos que constituyen nuestro patrimonio, pues forman un legado material que permite reconstruir el pasado de forma crítica y tangible, facilitando la comprensión de nuestro presente.

- Valorar la posesión de un patrimonio colectivo y común así como su conservación, disfrute y difusión.

- Hacer comprensible el patrimonio histórico y cultural mediante una interpretación científica y rigurosa al tiempo que motivadora.

Los contenidos aquí están estructurados en siete temas con una parte teórica y otra práctica en la que es esencial el trabajo autónomo ya que contamos solo con 2 horas de teoría y una de prácticas a la semana. La nota final es el resultado de la evaluación con una proporcionalidad del $50 \%$ cada una. Así, en clases teóricas tomamos como punto de partida el concepto de patrimonio y su evolución, el marco legal y la clasificación del patrimonio, y en seminarios consideramos interesante trabajar de forma más específica el patrimonio inmaterial.

El paso siguiente fue decidir la metodología íbamos a utilizar. Cómo docentes somos conscientes de que no existe una metodología única y universal válida para todo (García, M.P. 2014: p. 106), por ello, y teniendo en cuenta las problemáticas antes expuestas, tomamos la decisión de combinar diferentes metodologías adaptadas al desarrollo de la experiencia, ya que, no olvidamos que el aprendizaje es un proceso continuo de construcción y para lograrlo necesitamos que los/as alumnos/as experimenten toda la variedad posible de métodos (Dale, 1946; Dewey, 1916).

Debía ser, por tanto, una metodología que permitiera a los/as alumno/as trabajar sobre un tema de su interés, favoreciendo la investigación, el trabajo en grupo, la resolución de problemas, el aumento de las habilidades sociales y de comunicación, así como de la autoestima, aprovechando las habilidades de cada uno de los miembros del grupo desde la motivación y con un carácter lúdico.

Durante las sesiones teóricas utilizamos una metodología activa y participativa en las que nos servimos del método de observación directa con salidas al exterior ${ }^{8}$. En la parte práctica usamos el trabajo por proyectos con el que su precursor Kilpatrick pretendía que la enseñanza-aprendizaje se convirtiera en una investigación básica estructurada en tres grandes etapas: inicio (surge ante el interés de los alumnos por resolver una situación o un tema); preparación-ejecución (proceso de documentación que tiene una doble finalidad: elaborar un material y hacerlo llegar a la comunidad) y

\footnotetext{
${ }^{8}$ En el temario de la asignatura hay tres temas referidos a la metodologia didáctica para el análisis de la arquitectura, la escultura y la pintura. Para que los alumnos alcancen el aprendizaje significativo los ponemos en contacto directo con bienes patrimoniales para que por observación directa partiendo de los materiales, soportes, plantas etc. observen y sepan leer los elementos tanto del patrimonio material como inmaterial.
} 
valoración (evalúa la satisfacción de las personas implicadas) (Kilpatrick, 1918: 319-335). Estas características dotan de gran interés a este modelo desde el punto de vista del patrimonio cultural, al poner en juego procedimientos específicos de la previsión espacial, temporal y de relación social, además enfrenta a los futuros maestros con problemas reales que tienen que resolver que proporcionan un extenso campo en temas de carácter social y cultural, la dificultad radicaba en elegir dentro del patrimonio inmaterial temas que fueran atractivos para nuestros alumnos, así fue como seleccionamos el patrimonio gastronómico. Organizados en grupos de cinco los estudiantes debían trabajar centrándose en los alimentos que se incorporaron a nuestra dieta en diferentes momentos de la historia. El tema tenía muchas posibilidades didácticas y para darle ese carácter lúdico y motivador decidimos complementar la metodología con las TIC como estrategia didáctica colaborando con ello también a que adquirieran la competencia digital. De todos los recursos que ofrecen en la actualidad las nuevas tecnologías elegimos la WebQuest.

\section{RAZONES DE LA UTILIZACIÓN DE LA WEBQUET COMO ESTREGIA DIDÁCTICA}

Para lograr los mejores resultados en la elección de las estrategias didácticas hay que pensar y definir para qué la vamos a usar; quién la desarrolla, dónde y cuándo y con qué recursos. La WebQuest era la herramienta que más se adaptaba a nuestras necesidades suponiendo una innovación utilizarla de manera conjunta con la metodología por observación directa y el trabajo por proyectos. Creada por Bernie Dodge en 1995 en la Universidad Estatal de San Diego ha sido muy utilizada en enseñanza primaria y secundaria pero poco en la universidad (Dodge, B. y otros 1998). Su uso posibilita una actividad de indagación/investigación enfocada a que los estudiantes obtengan toda o la mayor parte de la información que van a utilizar de recursos existentes en Internet; es una exploración dirigida, que finaliza con la producción de una Web en la que se publica el resultado de una investigación, por tanto, es una metodología de aprendizaje basada en los recursos proporcionados por Internet que incitan a los alumnos a investigar, a potenciar el pensamiento crítico, la creatividad y la toma de decisiones al tiempo que contribuyen a desarrollar diferentes capacidades, llevando al alumnado a transformar los conocimientos adquiridos evitando la denostada costumbre de "copiar y pegar". Como es sabido consta de seis partes esenciales: Introducción, Tarea, Proceso, Recursos, Evaluación y Conclusión. Con ella se solucionaba una de las dificultades que presenta el trabajo por proyectos, pues a veces la investigación necesita de un tiempo del que no disponíamos. Con la WebQuest se soluciona pues uno de sus objetivos son que los alumnos/as haga un buen uso del tiempo; que la investigación se centre en utilizar información más que en buscarla y en apoyar el desarrollo de su pensamiento en los niveles de análisis, síntesis y evaluación.

\section{RESULTADOS}


Los resultados han sido muy satisfactorios. Los/as alumnos/as, que al inicio de las clases consideraban el patrimonio como algo lejano relacionado más con el ocio y el tiempo libre que con la educación y poco interesante para los niños de primaria, al finalizar el curso habían aprendido a apreciar su valor educativo en relación a la importancia de la contextualización espacial y del conocimiento del tiempo histórico a través de él. Además en la realización de los trabajos propuestos en los seminarios se desarrolló una corriente de superación e interés que hizo que cada grupo se esforzara por lograr que su trabajo fuera el mejor. Como temáticas específicas finalmente se abordó el chocolate y materias prima como el aceite de oliva, el tomate o la berenjena; platos tradicionales de la gastronomía andaluza como el ajoblanco, el salmorejo o el gazpacho y repostería española muy diversa. Fueron un total de unos 58 trabajos por curso estructurados en forma de WebQuest de los cuales el $80 \%$ alcanzaron el sobresaliente, el $15 \%$ notable, $3 \%$ aprobado y un $2 \%$ suspenso, con los que los estudiantes demostraron que habían logrado los objetivos de forma activa, participativa, autónoma y lúdica.

Nuestra propuesta metodológica utilizando de forma complementaria el trabajo por proyectos y la WebQuest ha supuesto una innovación que ha mostrado su utilidad para que los/as alumnos/as en formación logren las competencias establecidas en la Guía Docente así como su aprendizaje significativo. Además (y a modo de conclusión):

- Se ha mejorado la calidad docente.

- Se ha logrado que los estudiantes tomen conciencia y aprecien la importancia de conocer la sociedad en la que viven, adquieran instrumentos para acercarse a las raíces históricas de las sociedades actuales y pasadas, conozcan las diferentes manifestaciones culturales y valoren su diversidad.

- Que adquieran conocimientos sobre aspectos espaciales y sobre la evolución del pensamiento, las costumbres, las creencias y los movimientos sociales y políticos a lo largo de la historia.

- Desarrollen el interés y el respeto por el patrimonio cultural, tanto material como inmaterial.

- Conozcan la existencia de las posibilidades educativas de las TIC en la enseñanza-aprendizaje del patrimonio y

- Reconozcan la importancia del trabajo en grupo.

\section{REFERENCIAS}

DALE, E. (1946). Audiovisual methods in teaching. Nueva York: The Dryde Press.

DEWEY, J. (1916). Democracy and education: an intruduction to the philosophy of education. Nueva York: The Macmillan Company.

DODGE, B. y otros (1998). The WebQuest Page. San Diego: Departamento de Tecnología Educativa. Universidad de San Diego. Disponible en: htpp://WebQuest.sdsu.edu/.

GALLEGO, J. L. y Mata, F. S. (2005). Metodología de la acción didáctica. En: A. Medina y 
F.S. Mata (coord.), Didáctica General. Madrid. Prentice-Hall, PP. 221-245.

GARCÍA, M. P. (2014). Estrategias didácticas. Modos de enseñar y aprender. En: Gómez, I. y García, F. J. (Coord). Manual de Didáctica. Aprender a Enseñar. Madrid: Pirámide, 2014, pp. 98-119.

GONZÁLEZ, N. (2008) Una investigación cualitativa y etnográfica sobre el valor educativo y el uso didáctico del patrimonio cultural. En: Enseñanza de la Ciencias Sociales, 7. P. 24.

Ley de Educación de Andalucía, de 17/2007, 10 de noviembre.

Ley Orgánica de Educación 2006, 3 de Mayo.

Kilpatrick, W.H. (1918). The project method. Teachers College Record, 19.

Real Decreto 1513/2006, de 7 de diciembre por el que se establecen las enseñanzas mínimas en Educación Primaría.

Real Decreto 230/2007, de 31 de Julio, por el que se establece la ordenación y las enseñanzas correspondientes a la educación primaria en Andalucía.

\section{Para citar este artículo:}

Cambil, M. E. \& Romero, G. (2015). La WebQuest: una estrategia didáctica en el aula para la enseñanza-aprendizaje del patrimonio cultural. EDUTEC, Revista Electrónica de Tecnología Educativa, 52. Recuperado el dd/mm/aa de http://www.edutec.es/revista 